\section{Infecções sexualmente transmissíveis em mulheres afrodescendentes de comunidades quilombolas no Brasil: prevalência e fatores associados}

\author{
Sexually transmissible infections in African- \\ descendant women in maroon communities \\ in Brazil: prevalence and associated factors
}

\section{Enfermedades de transmisión sexual en mujeres afrodescendientes de comunidades quilombolas en Brasil: prevalencia y factores asociados}

\section{Resumo}

O objetivo do estudo foi estimar a prevalência de infecções sexualmente transmissíveis (IST) e fatores associados sobre mulheres quilombolas no Brasil. Trata-se de estudo transversal de base populacional com mulheres quilombolas no período de março de 2017 a janeiro de 2019. Utilizou-se um questionário com informações sociodemográficas, comportamentais e clínicas. Foi realizado exame ginecológico para coleta de células cervicais para citologia oncótica e para detecção de Chlamydia trachomatis, Neisseria gonorrhoeae, Trichomonas vaginalis e papilomavírus humano (HPV), por meio de teste de reação em cadeia pela polimerase. Foi realizado teste rápido para HIV e sífilis. $O$ desfecho principal foi definido como a infecção por um ou mais agentes infecciosos de transmissão sexual. Para a análise, utilizou-se o teste do quiquadrado e regressão logística hierárquica. De um total de 380 mulheres, 352 (92,6\%) foram incluídas no estudo. A prevalência de, pelo menos, uma IST foi de 18,5\% (IC95\%: 14,76-22,85). A maior prevalência foi de 11,1\% por HPV, seguido de 6,3\% por $\mathrm{T}$. vaginalis e de 4,3\% por C. trachomatis. Não houve nenhum caso de N. gonorrhoeae. Para o HIV, a prevalência foi de 0,3\%, e de sifilis foi de 4,3\%. A citologia cervicovaginal estava alterada em 7,7\%. A detecção de uma ou mais IST foi significativamente associada a idade entre 25 e 44 anos (OR = 2,33; IC95\%: 1,05-5,18), a consumo de álcool (OR = 1,96; IC95\%: 1,06-3,64), a resultado alterado da citologia $(O R=3,96 ;$ IC95\%: 1,65-9,48) e a vaginose bacteriana $(O R=3,61$; IC95\%: 2,01-6,47). Em mulheres quilombolas houve elevada prevalência de uma ou mais IST, o que torna importante a elaboração de estratégias de prevenção direcionadas a essas mulheres.

Doenças Sexualmente Transmissíveis; Mulheres; Estudos Transversais
Jerusa Araujo Dias 1

Thais Verly Luciano 2

Maria Carmen Lopes Ferreira Silva Santos 2

Carlos Musso ${ }^{2}$

Eliana Zandonade 2

Liliana Cruz Spano 2

Angelica Espinosa Miranda 2

doi: 10.1590/0102-311X00174919

\section{Correspondência}

J. A. Dias

Universidade Federal do Espirito Santo.

Rod. Governador Mario Covas Km 60 Norte, São Mateus, ES 29932-540, Brasil.

dias.saude@gmail.com

1 Universidade Federal do Espírito Santo, São Mateus, Brasil.

2 Universidade Federal do Espírito Santo, Vitória, Brasil. 


\section{Introdução}

As infecções sexualmente transmissíveis (IST) causadas por Chlamydia trachomatis, Neisseria gonorrhoeae, Trichomonas vaginalis e pelo Treponema pallidum, causador da sífilis, são consideradas curáveis pela Organização Mundial da Saúde (OMS) 1. Somadas, infectaram aproximadamente 500 milhões de pessoas entre 15 e 49 anos em todo o mundo, no ano de 2015 1,2. Um total de 126 milhões de novos casos ocorreu nos países do continente americano, sendo 7,6\% para C. trachomatis, 0,8\% para N. gonorrhoeae e 22,0\% para T. vaginalis 1 . Em 2016, no mundo, havia mais de 600 mil gestantes com sífilis 2 . Os números de novos casos de sífilis adquirida e de sífilis congênita apresentaram uma ligeira queda, considerando o período de 2012 a 2015, porém esses números ainda permanecem elevados 1,2 .

As IST de etiologia viral mais prevalentes são as causadas pelo papilomavírus humano (HPV) e pelo vírus da imunodeficiência humana (HIV) 1,3. A infecção viral pelo HPV foi responsável, em 2015, por 15 milhões de infecções em mulheres de todo o mundo, com mais de 600 mil casos no continente americano ${ }^{3}$. Por outro lado, o número de pessoas no mundo que vivia com o HIV, em 2017, foi de quase 37 milhões, sendo 3,4 milhões nas Américas 4.

Segundo estudos internacionais, as IST são responsáveis por vários desfechos negativos relacionados à gravidez e à saúde sexual e reprodutiva das mulheres, como prematuridade 5 , ruptura precoce das membranas 6 , baixo peso ao nascer 5,6 , infertilidade 7 , câncer de colo de útero 7,8 , doença inflamatória pélvica 9 , além de aumento do risco de infecção pelo HIV 10. Elas são mais frequentes em mulheres negras 11,12, e a maioria dos casos é assintomático 13.

As mulheres negras no Brasil apresentam situações de vulnerabilidade que as fragilizam nas questões relacionados a morbidade, acesso e atenção à saúde, principalmente nos aspectos relacionados à saúde sexual e reprodutiva 14,15. Nesse contexto de desigualdades e vulnerabilidades, estão inseridas as comunidades quilombolas. Essas comunidades são afrodescendentes, tradicionais e contam com uma forma própria de organização social e reprodução de hábitos culturais e ancestralidade relacionados a uma resistência história de direitos sociais e econômicos 16.

Diferenças étnico-raciais relacionados às IST são frequentemente objeto de estudo nos países da América do Norte 17,18 e da África 19,20, mas no Brasil são escassas as pesquisas que abordam essa temática, principalmente quando se trata de mulheres negras e de população rural 21. Não existem informações publicadas sobre a saúde sexual e reprodutiva de mulheres quilombolas no Estado do Espírito Santo, e os estudos atuais disponíveis no Brasil foram realizados em populações quilombolas da Região Nordeste do país 22,23.

Logo, as informações sobre dados comportamentais e clínicos sobre as IST em mulheres afrodescendentes de comunidades quilombolas contribuirão com a ampliação do conhecimento sobre o perfil epidemiológico dessas infecções nessa população, gerando subsídios para o debate acerca da Política Nacional de Saúde Integral da População Negra (PNSIPN), para a implementação de políticas públicas baseadas em evidências e na elaboração de dados robustos para futuras revisões sistemáticas. Assim, o presente estudo tem como objetivo estimar a prevalência e os fatores associados às IST em mulheres afrodescendentes de comunidades quilombolas da Região do Sapê do Norte, Espírito Santo.

\section{Material e métodos}

Realizou-se um estudo transversal de base populacional por meio de uma amostragem não probabilística, na qual foram incluídas as mulheres quilombolas em suas comunidades de origem. Optou-se por essa amostragem, pois as mulheres não estavam vinculadas a nenhum serviço público de assistência a saúde, educação ou assistência social, o que dificultou a amostragem por grupo de domicílios e/ou por faixa etária. Os pesquisadores, após contato com as lideranças comunitárias, foram até as comunidades para convidar as mulheres quilombolas.

O estudo foi conduzido entre março de 2017 e fevereiro de 2019, com mulheres autodeclaradas quilombolas, integrantes da chamada Região do Sapê do Norte, área quilombola localizada entre os municípios de São Mateus e Conceição da Barra, no norte do Espírito Santo (Figura 1).

As mulheres que já haviam iniciado a vida sexual, residentes nas comunidades quilombolas da área rural ou urbana, foram consideradas elegíveis para o estudo. As participantes foram convidadas 


\section{Figura 1}

Mapa das comunidades quilombolas da Região do Sapê do Norte, municípios de São Mateus e Conceição da Barra, norte do Espírito Santo, Brasil, 2017-2019.
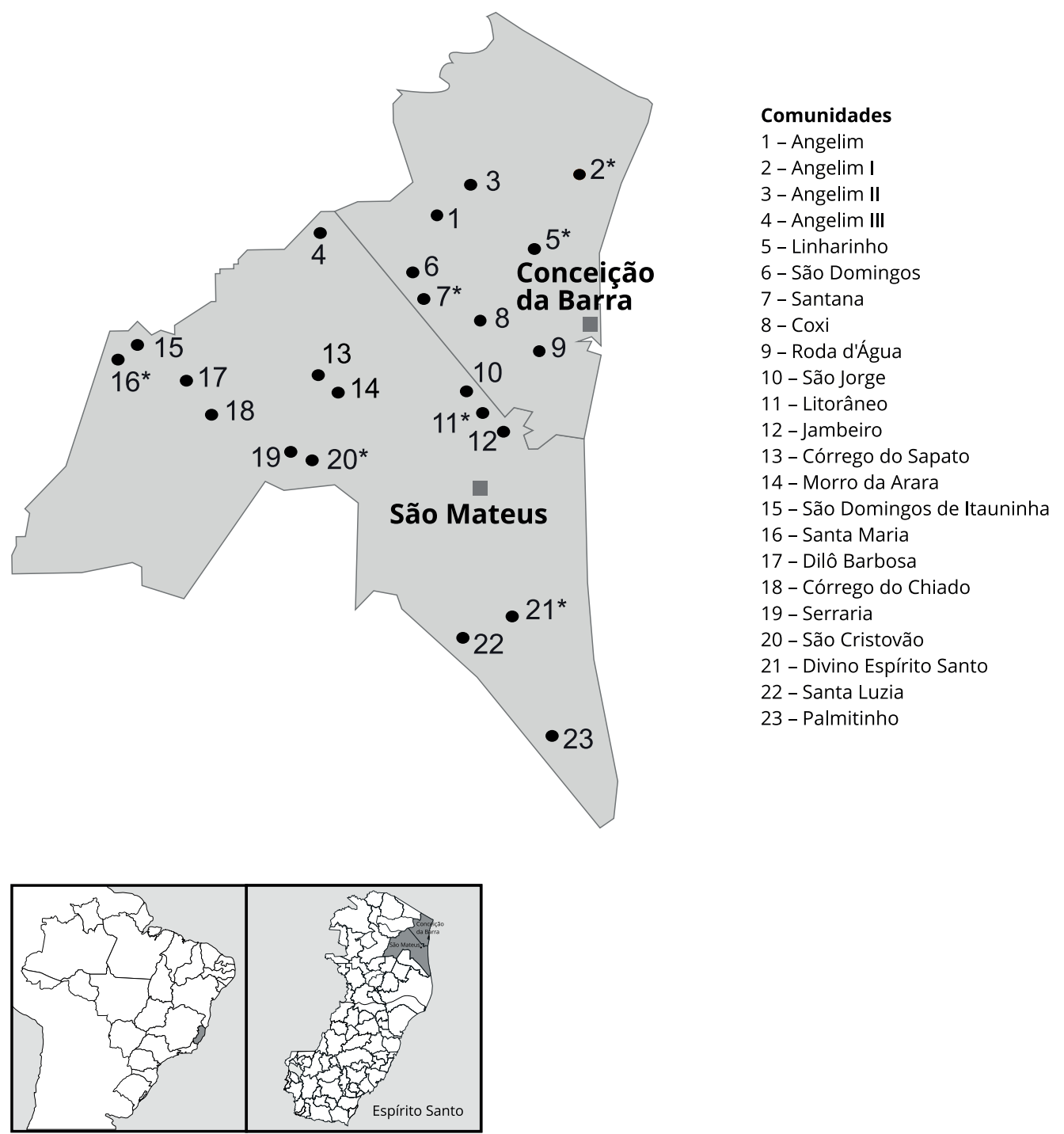

Fonte: elaboração própria.

* Comunidades que possuem Estratégia Saúde da Família (ESF).

a responder uma entrevista face a face, contendo informações sociodemográficos e comportamentais (idade, local de residência, escolaridade, uso de álcool e uso de drogas ilícitas), comportamentos sexuais e características reprodutivas (uso de método contraceptivo, uso de preservativo com parceiro, coitarca antes de 15 anos, idade do parceiro na ocasião da primeira relação sexual da mulher, número de parceiros sexuais na vida e nos últimos 12 meses) e clínicas (história de IST, testagem anti-HIV, realização do último exame citopatológico, presença de sintomas ginecológicos, resultado do último exame citopatológico, diagnóstico de vaginose bacteriana). 
As entrevistas foram realizadas em local privativo de escolha da participante, a fim de garantir a validade das respostas e de assegurar o sigilo da participação no estudo. Nesse mesmo local, foi realizado o exame ginecológico para a coleta do exame citopatológico e para a coleta de espécimes dos demais agentes infecciosos. As pesquisadoras responsáveis pelos procedimentos participaram de reuniões com as lideranças quilombolas da Região do Sapê do Norte e ofertaram oficinas de educação em saúde para as mulheres da comunidade. Elas também tinham experiência anterior como enfermeiras nas unidades básicas de saúde do município, fato que contribuiu para a formação de laços de confiança com a comunidade.

Não foram incluídas no estudo as mulheres que não haviam iniciado a vida sexual, as gestantes e as mulheres que não tinham ascendência quilombola ou que não se autodeclararam quilombola. O resultado do exame citopatológico obedeceu à Nomenclatura Brasileira para Laudos Citopatológicos Cervicais 24 do Ministério da Saúde. Também foi oferecida testagem rápida para diagnóstico de HIV e de sífilis às participantes.

Aproximadamente duas semanas após a entrevista e a coleta de espécimes, foi marcada consulta de retorno, com os objetivos de devolução do resultado do exame e de realização do tratamento da mulher e do parceiro, se necessário. Na oportunidade, aconselhava-se como prevenir as IST e o câncer de colo de útero. Os casos com resultados contendo alterações de lesões cervicais de alto grau foram encaminhados ao Serviço de Ginecologia do Hospital Universitário Cassiano Antônio de Moraes (HUCAM), da Universidade Federal do Espírito Santo (UFES), para tratamento adequado. As mulheres com exame citopatológico que apresentaram lesões cervicais de baixo grau foram encaminhadas para acompanhamento pelas unidades de saúde do município de residência da mulher quilombola, com a garantia do acompanhamento.

O desfecho principal, infecção genital (não detectado; detectado), foi definido como a detecção, por meio do teste de PCR ou PCR em tempo real, de IST por um ou mais agentes: C. trachomatis, T. vaginalis, $N$. gonorrhoeae e HPV. Para a infecção pelo HIV, a detecção foi realizada por meio do teste rápido. Para a detecção da sífilis, em caso positivo no teste rápido, foi realizado o teste VDRL [Veneral Disease Research Laboratory].

O universo amostral estimado baseou-se em 1.200 famílias quilombolas cadastradas pela Fundação Cultural Palmares 25. O cálculo amostral foi necessário para evitar a realização do censo, o que diminuiu os custos e garantiu representatividade da população. Para o cálculo do tamanho da amostra, foi considerada uma taxa de 3,8\% de alterações celulares de colo de útero em mulheres no Brasil 26, com nível de $95 \%$ de confiança, variabilidade de $\pm 2 \%$, poder de $80 \%$ e nível de significância menor que 5\%. Esse cálculo resultou em um total de 336 mulheres. Supondo-se uma perda de 10\%, o tamanho final da amostra foi definido em no mínimo 370 mulheres.

Foi realizada análise descritiva, incluindo distribuição de frequência para variáveis qualitativas, assim como cálculo de média, mediana e desvio padrão (DP) e intervalo interquartil (IQT) para variáveis quantitativas. Para verificar a associação entre as características sociodemográficas, sexuais, reprodutivas e clínicas, foi realizado o teste do qui-quadrado com correção de Yates ou teste exato de Fisher, quando apropriado. Odds ratio (OR) e intervalos de 95\% de confiança (IC95\%) foram obtidos na análise. Foi realizada análise bivariada, utilizando-se o teste de qui-quadrado de Pearson, entre as variáveis do mesmo nível para determinação do valor de significância estatística e para seleção das variáveis que seriam incluídas no modelo de regressão múltipla.

Para estimar o efeito de uma variável, ao mesmo tempo em que se controla o efeito das demais na probabilidade de ter IST, foi utilizada regressão logística hierarquizada. As variáveis preditoras foram hierarquicamente inseridas em três blocos distintos: bloco proximal (sintomas ginecológicos, dor pélvica, sangramentos genitais frequentes, coceira vaginal, corrimento vaginal, adenopatia inguinal, ardência ao urinar, ferida genital, tempo de realização do último preventivo, resultado do último preventivo, detecção de vaginose bacteriana), bloco intermediário (consumo de álcool, consumo de droga ilícita, uso de método contraceptivo, uso de preservativo com parceiro, coitarca antes dos 15 anos, idade do parceiro na ocasião da coitarca da mulher, relação sexual anal, número de parceiros sexuais na vida, número de parceiros sexuais nos últimos 12 meses, história de IST, testagem para HIV) e bloco distal (idade, renda, escolaridade, local de residência).

Foi utilizado o método forward para seleção das variáveis. A análise de regressão logística hierarquizada foi iniciada pelo nível distal, seguido do intermediário, até o nível proximal. As variáveis de 
cada bloco foram ajustadas pelas demais variáveis significativas do mesmo nível e por aquelas retidas nos níveis hierárquicos superiores.

As variáveis inseridas no modelo hierárquico foram as que apresentaram um valor de $\mathrm{p} \leq 0,10$ na etapa da análise bivariada, tendo permanecido no modelo final de análise múltipla aquelas com valor de $\mathrm{p} \leq 0,05$. A escolha do modelo hierarquizado para análise dos dados seguiu a proposta de Victora et al. 27 , uma vez que essa técnica contribui com a interpretação dos resultados de estudos epidemiológicos que contam com uma estrutura complexa de inter-relação entre as variáveis independentes com o desfecho de interesse, levando em consideração o conhecimento social e biológico disponível na literatura científica. Todas as informações foram codificadas e armazenadas anonimamente em um banco de dados criado para esse fim. Para a análise dos dados, foi utilizado o programa estatístico SPSS, versão 21.0 (https://www.ibm.com/).

Este estudo foi submetido ao Comitê de Ética em Pesquisa do Centro de Ciências da Saúde da UFES, sendo aprovado, sob os números do Certificado de Apresentação para Apreciação Ética (CAAE) 44205714.1.0000.5060 e 01218818.3.0000.5060, de 4 de novembro de 2015 e 11 de dezembro de 2018, respectivamente. Todas as mulheres quilombolas que aceitaram participar do estudo assinaram um termo de consentimento livre e esclarecido (TCLE).

\section{Resultados}

Um total de 380 mulheres quilombolas foi convidado a participar do estudo. Dezoito mulheres foram consideradas não elegíveis, uma vez que não se autodeclararam quilombolas (estavam vinculadas à comunidade por matrimônio), e dez mulheres não concordaram em assinar o TCLE. Após as perdas, a amostra selecionada foi de 352 mulheres, conforme Figura 2. A prevalência geral de IST foi de 18,5\%,

\section{Figura 2}

Fluxograma de seleção das mulheres quilombolas da Região do Sapê do Norte, Espírito Santo, Brasil, 2017-2019.

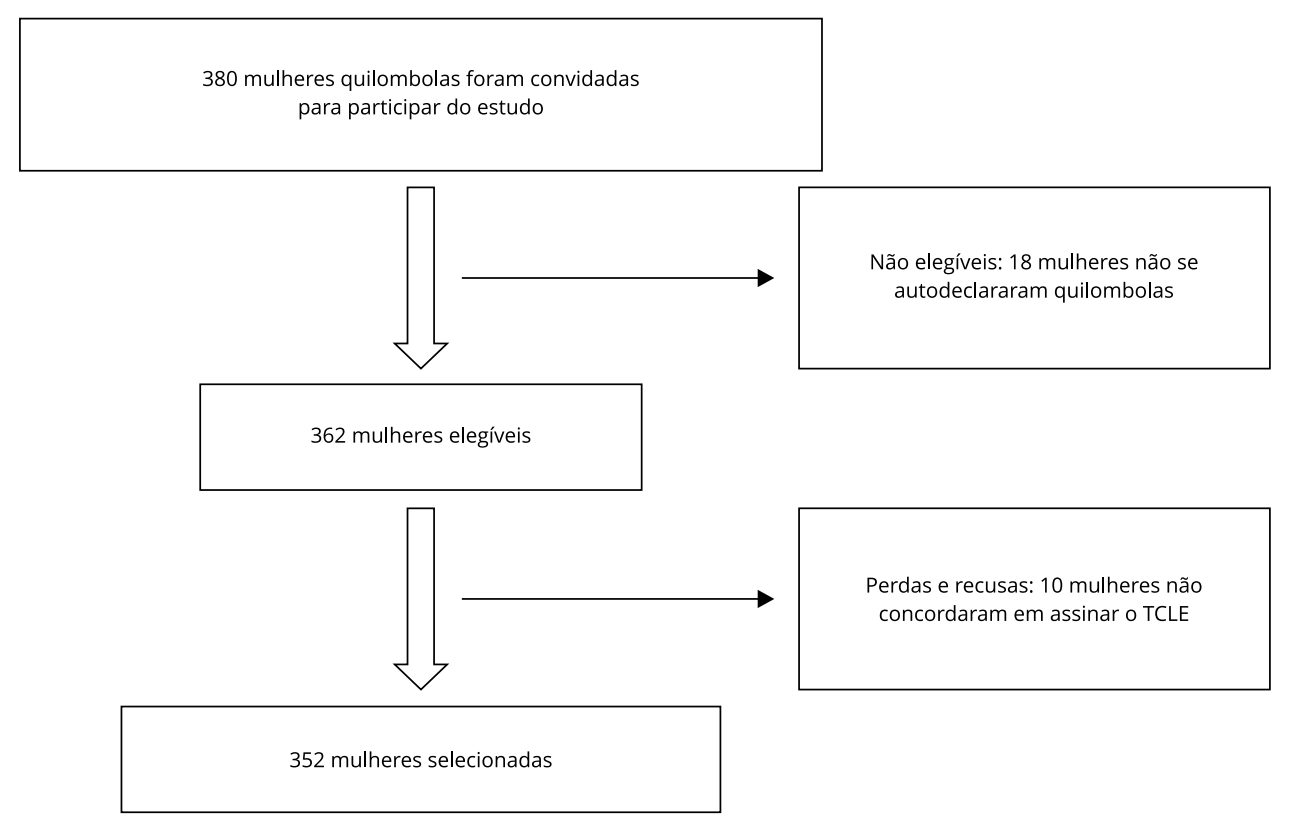

TCLE: termo de consentimento livre e esclarecido. 
com maior prevalência encontrada para HPV (11,1\%), seguida por T. vaginalis (6,3\%), C. trachomatis $(4,3 \%)$ e T. pallidum (4,3\%). Nenhum caso de infecção por $N$. gonorrhoeae foi detectado. Infecção por HIV foi de 0,3\% (Tabela 1). Coinfecção entre C. trachomatis e T. vaginalis foi observada em $0,8 \%$ dos casos (IC95\%: 0,29-2,4).

A média de idade das mulheres quilombolas foi de 41,4 anos ( $D P=14,3$ anos) e, para a idade da primeira relação sexual, a média foi de 16,7 anos ( $(\mathrm{DP}=3,1$ anos). A mediana de idade do parceiro sexual na primeira relação sexual da mulher foi de 20 anos (mínimo de 15 anos e máximo de 52 anos).

A distribuição por faixa etária da amostra revelou maior frequência de mulheres quilombolas com idade entre 25 e 44 anos (45,2\%). A proporção de mulheres quilombolas com renda menor ou igual a $\mathrm{R} \$ 406,00$ foi de $56,3 \%$. Do total, $83,8 \%$ residiam na área rural e $67,9 \%$ tinham menos de oito anos de escolaridade. O difícil acesso aos serviços de saúde foi mencionado por $61,5 \%$ das mulheres quilombolas, conforme Tabela 2 . O consumo de álcool foi relatado por $25,6 \%$ das mulheres quilombolas (Tabela 2).

As características sexuais das mulheres quilombolas mostraram que 66,5\% faziam uso de algum método contraceptivo e $78,7 \%$ não usavam preservativo com parceiro (Tabela 2). Para 39,1\% das mulheres, a primeira relação sexual ocorreu com menos de 15 anos de idade. A prática de relação sexual anal foi de $25,3 \%$. Um grupo de $24,5 \%$ das mulheres indicou ter se relacionado com cinco ou mais parceiros sexuais na vida, ao passo que $5,1 \%$ das entrevistadas relataram dois ou mais parceiros sexuais nos últimos 12 meses (Tabela 2).

Do total de mulheres, $4,3 \%$ citaram história de IST e 12,2\% nunca tinham feito testagem anti-HIV. Queixas de sintomas ginecológicos foram relatadas por 59,1\% (Tabela 2).

A prevalência geral de alteração no exame citopatológico foi de 7,7\%, sendo a lesão de baixo grau (LSIL) a mais frequente, com $6 \%$ de prevalência. A prevalência de vaginose bacteriana foi de $31,8 \%$ (Tabela 2).

$\mathrm{Na}$ análise bivariada, as variáveis associadas a infecção genital com valor de $\mathrm{p}<0,10$ foram as seguintes: idade, uso de álcool, resultado do exame citopatológico e vaginose bacteriana. Após o ajuste no modelo de regressão múltipla, as variáveis independentes que estiveram associadas a, pelo menos, uma IST foram as seguintes: idade entre 25 a 44 anos (OR = 2,01; IC95\%: 1,07-5,89), consumo de álcool (OR = 1,96; IC95\%: 1,06-3,64), exame citopatológico alterado (OR = 3,96; IC95\%: 1,65-9,48) e diagnóstico de vaginose bacteriana (OR = 3,61; IC95\%: 2,01-6,47) (Tabela 3).

Tabela 1

Prevalência geral de infecção genital, infecção por Chlamydia trachomatis, por Trichomonas vaginalis, HPV, vaginose bacteriana, HIV e por Treponema pallidum em mulheres quilombolas da Região do Sapê do Norte, Espírito Santo, Brasil, 2017-2019.

\begin{tabular}{lccc}
\hline Infecção sexual & $\mathbf{n}$ & $\%$ & IC95\% \\
\hline Infecção genital * & 65 & 18,5 & $14,76-22,85$ \\
C. trachomatis ** & 15 & 4,3 & $2,60-6,91$ \\
T. vaginalis ** & 22 & 6,3 & $4,16-9,28$ \\
HPV (qualquer tipo) ** & 39 & 11,1 & $8,21-14,79$ \\
Vaginose bacteriana & 112 & 31,8 & $27,17-58,37$ \\
HIV & 1 & 0,3 & $0,05-1,59$ \\
T. pallidum & 15 & 4,3 & $2,60-6,91$ \\
\hline
\end{tabular}

IC95\%: intervalo de 95\% de confiança.

* Desfecho principal. Pode ser considerada uma ou mais infecções sexuais;

** Infecção sexual utilizada para classificar a infecção genital. 


\section{Tabela 2}

Análise bruta das características sociodemográficas, comportamentais e clínicas associadas a infecção genital em mulheres quilombolas da Região do Sapê do Norte, Espírito Santo, Brasil, 2017-2019.

\begin{tabular}{|c|c|c|c|c|}
\hline Características sociodemográficas, comportamentais e clínicas & $\mathbf{N}(\%)$ & $\begin{array}{l}\text { Detectada } \\
\text { n (\%) }\end{array}$ & $\begin{array}{l}\text { OR bruto } \\
\text { (IC95\%) }\end{array}$ & Valor de $p$ * \\
\hline \multicolumn{5}{|l|}{ Bloco distal } \\
\hline Idade (anos) & & & & 0,04 \\
\hline $14-24$ & $39(11,10)$ & $13(33,33)$ & 1,00 & \\
\hline $25-44$ & $159(45,20)$ & $26(16,35)$ & $2,46(1,11-5,41)$ & \\
\hline 45 e mais & $154(43,80)$ & $26(16,88)$ & $0,96(0,53-1,74)$ & \\
\hline Renda (Reais) ** & & & & 0,66 \\
\hline$>406,00$ & $154(43,80)$ & $30(19,50)$ & 1,00 & \\
\hline$\leq 406,00$ & $198(56,30)$ & $35(17,70)$ & $1,12(0,65-1,93)$ & \\
\hline Zona de residência & & & & 0,20 \\
\hline Urbana & $57(16,20)$ & $14(24,56)$ & 1,00 & \\
\hline Rural & $295(83,80)$ & $51(17,29)$ & $0,64(0,32-1,26)$ & \\
\hline Anos de estudo & & & & 0,13 \\
\hline$>8$ & $113(32,10)$ & $26(23,01)$ & 1,00 & \\
\hline$\leq 8$ & $239(67,90)$ & $39(16,32)$ & $1,53(0,87-2,67)$ & \\
\hline Acesso ao serviço de saúde *** & & & & 0,77 \\
\hline Fácil & $135(38,50)$ & $24(17,80)$ & 1,00 & \\
\hline Difícil/Impossível & $216(61,50)$ & $41(19,00)$ & $1,08(0,62-1,89)$ & \\
\hline \multicolumn{5}{|l|}{ Bloco intermediário } \\
\hline Uso de álcool & & & & 0,00 \\
\hline Não & $262(74,40)$ & $40(15,27)$ & 1,00 & \\
\hline Sim & $90(25,60)$ & $25(27,78)$ & $2,13(1,20-3,77)$ & \\
\hline Uso de droga & & & & 0,36 \\
\hline Não & $340(96,60)$ & $64(18,82)$ & 1,00 & \\
\hline Sim & $12(3,40)$ & $1(8,33)$ & $2,55(0,32-20,11)$ & \\
\hline Uso de método contraceptivo & & & & 0,60 \\
\hline Não & $118(33,50)$ & $20(16,95)$ & 1,00 & \\
\hline Sim & $234(66,50)$ & $45(19,23)$ & $0,85(0,48-1,53)$ & \\
\hline Uso de preservativo com parceiro & & & & 0,06 \\
\hline Não & $273(78,70)$ & $44(16,12)$ & 1,00 & \\
\hline Sim & $74(21,30)$ & $19(25,68)$ & $1,79(0,97-3,32)$ & \\
\hline Idade da coitarca (anos) & & & & 0,66 \\
\hline 15 ou mais & $213(60,90)$ & $38(17,84)$ & 1,00 & \\
\hline Antes de 15 & $137(39,10)$ & $27(19,71)$ & $1,13(0,65-1,95)$ & \\
\hline Idade do parceiro sexual na ocasião da coitarca da mulher (anos) & & & & 0,46 \\
\hline$\leq 20$ & $139(48,80)$ & $23(16,55)$ & 1,00 & \\
\hline$>20$ & $146(51,20)$ & $29(19,86)$ & $1,25(0,68-2,28)$ & \\
\hline Número de parceiros sexuais na vida & & & & 0,98 \\
\hline $1-4$ & $265(75,50)$ & $49(18,49)$ & 1,00 & \\
\hline 5 ou mais & $86(24,50)$ & $16(18,60)$ & $1,00(0,53-1,88)$ & \\
\hline Relação sexual anal & & & & 0,65 \\
\hline Não & $263(74,70)$ & $50(19,01)$ & 1,00 & \\
\hline Sim & $89(25,30)$ & $15(16,85)$ & $1,15(0,61-2,18)$ & \\
\hline Número de parceiros sexuais nos últimos 12 meses & & & & 0,22 \\
\hline 0 ou 1 & $298(84,90)$ & $52(17,45)$ & 1,00 & \\
\hline 2 ou mais & $53(15,10)$ & $13(24,53)$ & $1,53(0,76-3,07)$ & \\
\hline
\end{tabular}

(continua) 
Tabela 2 (continuação)

\begin{tabular}{|c|c|c|c|c|}
\hline Características sociodemográficas, comportamentais e clínicas & $\mathbf{N}(\%)$ & $\begin{array}{c}\text { Detectada } \\
\text { n (\%) }\end{array}$ & $\begin{array}{l}\text { OR bruto } \\
\text { (IC95\%) }\end{array}$ & Valor de $p$ * \\
\hline História de IST & & & & 0,60 \\
\hline Não & $337(95,70)$ & $63(18,69)$ & 1,00 & \\
\hline Sim & $15(4,30)$ & $2(13,33)$ & $1,49(0,32-6,79)$ & \\
\hline Fez teste anti HIV? & & & & 0,91 \\
\hline Não & $117(12,20)$ & $22(18,80)$ & 1,00 & \\
\hline Sim & $235(33,20)$ & $43(18,30)$ & $0,96(0,54-1,70)$ & \\
\hline \multicolumn{5}{|l|}{ Bloco proximal } \\
\hline Sintomas ginecológicos & & & & 0,50 \\
\hline Não & $144(40,90)$ & $29(20,14)$ & 1,00 & \\
\hline Sim & $208(59,10)$ & $36(17,31)$ & $1,20(0,70-2,07)$ & \\
\hline Dor pélvica & & & & 0,55 \\
\hline Não & $216(61,40)$ & $42(19,44)$ & 1,00 & \\
\hline Sim & $136(38,60)$ & $23(16,91)$ & $1,18(0,67-2,07)$ & \\
\hline Sangramento genital & & & & 0,84 \\
\hline Não & $334(94,90)$ & $62(18,56)$ & 1,00 & \\
\hline Sim & $18(5,10)$ & $3(16,67)$ & $1,14(0,32-4,05)$ & \\
\hline Coceira vaginal & & & & 0,68 \\
\hline Não & $247(70,20)$ & $47(19,03)$ & 1,00 & \\
\hline Sim & $105(29,80)$ & $18(17,14)$ & $1,13(0,62-2,06)$ & \\
\hline Corrimento vaginal & & & & 0,06 \\
\hline Não & $260(73,90)$ & $42(16,15)$ & 1,00 & \\
\hline Sim & $105(26,10)$ & $23(25,00)$ & $1,73(0,97-3,07)$ & \\
\hline Adenopatia inguinal & & & & 0,11 \\
\hline Não & $332(94,30)$ & $64(19,28)$ & 1,00 & \\
\hline Sim & $20(5,70)$ & $1(5,00)$ & $4,53(0,59-34,52)$ & \\
\hline Ardência ao urinar & & & & 0,24 \\
\hline Não & $279(79,30)$ & $55(19,71)$ & 1,00 & \\
\hline Sim & $73(20,70)$ & $10(13,70)$ & $1,54(0,74-3,20)$ & \\
\hline Ferida genital & & & & 0,87 \\
\hline Não & $340(96,60)$ & $63(18,53)$ & 1,00 & \\
\hline Sim & $12(3,40)$ & $2(16,67)$ & $1,13(0,24-5,31)$ & \\
\hline Resultado de exame citopatológico & & & & $<0,001$ \\
\hline Sem alterações & $325(92,30)$ & $51(15,69)$ & 1,00 & \\
\hline Alterado & $27(7,70)$ & $14(51,85)$ & $5,78(2,56-13,03)$ & \\
\hline Alterações do exame citopatológico & & & & 0,11 \\
\hline LSIL & $21(77,80)$ & $9(42,86)$ & 1,00 & \\
\hline HSIL & $6(22,20)$ & $5(83,33)$ & $6,66(0,65-67,46)$ & \\
\hline Vaginose bacteriana & & & & $<0,001$ \\
\hline Não detectado & $240(68,20)$ & $27(11,30)$ & 1,00 & \\
\hline Detectado & $112(31,80)$ & $38(33,90)$ & $4,05(2,31-7,09)$ & \\
\hline
\end{tabular}

HSIL: lesão intraepitelial escamosa de alto grau; IC95\%: intervalo de 95\% de confiança; LSIL: lesão intraepitelial escamosa de baixo grau; OR: odds ratio.

* Valor de p obtido pelo teste do qui-quadrado de Pearson;

** Renda individual da mulher quilombola;

*** Variável independente presente na tabela a título de informação ao leitor, pois não foi utilizada na análise hierarquizada. 


\section{Tabela 3}

Análise ajustada hierarquizada dos fatores associados a infecção genital em mulheres quilombolas da Região do Sapê do Norte, Espírito Santo, Brasil, 2017-2019.

\begin{tabular}{|c|c|c|c|c|c|c|}
\hline Variáveis independentes & $\begin{array}{l}\text { OR ajustado } \\
\text { (IC95\%)* }\end{array}$ & Valor de p & $\begin{array}{l}\text { OR ajustado } \\
\text { (IC95\%)** }\end{array}$ & Valor de p & $\begin{array}{l}\text { OR ajustado } \\
\text { (IC95\%)*** }\end{array}$ & Valor de $p$ \\
\hline \multicolumn{7}{|l|}{ Bloco distal } \\
\hline Idade (anos) & & 0,03 & & 0,05 & & 0,03 \\
\hline $14-24$ & 1,00 & & 1,00 & & 1,00 & \\
\hline $25-44$ & $2,33(1,05-5,18)$ & & $2,08(1,10-3,38)$ & & $2,01(1,07-5,89)$ & \\
\hline 45 e mais & $0,97(0,51-1,84)$ & & $0,83(0,32-2,13)$ & & $0,90(0,50-1,45)$ & \\
\hline \multicolumn{7}{|l|}{ Bloco intermediário } \\
\hline Uso de álcool & & & & 0,05 & & 0,03 \\
\hline Não & & & 1,00 & & 1,00 & \\
\hline Sim & & & $1,83(1,01-3,44)$ & & $1,96(1,06-3,64)$ & \\
\hline \multicolumn{7}{|l|}{ Bloco proximal } \\
\hline Resultado do exame citopatológico & & & & & & 0,00 \\
\hline Sem alterações & & & & & 1,00 & \\
\hline Alterado & & & & & $3,96(1,65-9,48)$ & \\
\hline Vaginose bacteriana & & & & & & $<0,001$ \\
\hline Não detectado & & & & & 1,00 & \\
\hline Detectado & & & & & $3,61(2,01-6,47)$ & \\
\hline
\end{tabular}

IC95\%: intervalo de 95\% de confiança; OR: odds ratio.

* Bloco distal;

** Bloco distal + Bloco intermediário;

*** Bloco distal + Bloco intermediário + Bloco proximal.

\section{Discussão}

Observamos neste estudo elevada prevalência geral (18,5\%) de uma ou mais IST na população de mulheres quilombolas da Região do Sapê do Norte. Entretanto, observamos menor prevalência por $C$. trachomatis, T. vaginalis e N. gonorrhoeae. Além disso, a presença de uma ou mais IST foi significativamente associada à idade da mulher, ao consumo de álcool, ao resultado alterado do exame citopatológico e ao diagnóstico de vaginose bacteriana. As mulheres negras têm risco aumentado para IST, HIV/ aids 12,17,18,28. A elevada prevalência geral de IST encontrada neste estudo foi maior que a descrita com mulheres da população em geral, residentes na área rural do Estado do Amazonas 29, bem como naquelas atendidas pela atenção primária no Estado de São Paulo 30.

Os estudos publicados no Brasil que abordaram investigação de múltiplas IST foram, em sua maioria, realizados em centros urbanos 31,32,33, em populações atendidas em clínicas especializadas 34,35. Poucos são os dados disponíveis sobre IST 23,36 em uma amostra de mulheres quilombolas, que vivem em sua maior parte em área rural, vulneráveis nos aspectos relacionados à saúde.

Particularmente em relação às infecções por C. trachomatis e N. gonorrhoeae, é desconhecida a real magnitude da carga dessas infecções na população, uma vez que não são de notificação compulsória 37 . Uma prevalência elevada dessas infecções, comparada aos nossos resultados, foi descrita em mulheres do interior do Amazonas 38 e na região central do país ${ }^{39}$. No entanto, semelhante ao encontrado neste estudo, uma baixa prevalência por $N$. gonorrhoeae foi descrita em mulheres na Região Centro-oeste 39 .

A prevalência de IST encontrada neste estudo abre uma janela de oportunidades para a realização de medidas de prevenção e de controle das IST na população quilombola, que vão desde a qualificação dos profissionais de saúde no aconselhamento e testagem rápida para as IST, educação em saúde e fornecimento de preservativos, até mesmo para os gestores de saúde, na implementação de políticas públicas direcionadas para essa população. 
A prevalência de infecção por qualquer tipo de HPV, neste estudo, foi semelhante à encontrada em mulheres de comunidades quilombolas no Maranhão e de uma outra comunidade quilombola no Nordeste do Brasil 28 . Porém, prevalência mais elevada foi encontrada em mulheres da área rural do Nordeste 40 .

Estudos 41,42,43 conduzidos com mulheres negras em outros países demonstraram que essa população apresenta um risco aumentado para a infecção pelo HPV e tem as maiores taxas de câncer de colo de útero 44,45, o que é explicado pelo difícil acesso a serviços de saúde, baixa renda, baixa escolaridade e pelo racismo institucional.

Esse fato é semelhante à elevada prevalência por HPV nas mulheres quilombolas deste estudo, uma vez que elas também têm baixa escolaridade, baixa renda e necessitam percorrer longas distâncias até o serviço de saúde mais próximo. A título de exemplo, a comunidade número 20 (Figura 1) encontra-se distante 13 quilômetros aproximadamente da unidade de saúde mais próxima, sendo esse trajeto percorrido por estrada rural, sem transporte público, de difícil acesso e sem asfaltamento. As comunidades quilombolas mais próximas do serviço de saúde são as de número 7 e 2 (Figura 1), que estão distantes 0,8 e 2,4 quilômetros, respectivamente, até o serviço de saúde mais próximo.

Como observado neste estudo, as dificuldades de acesso aos serviços de saúde ocorreram por dificuldades no transporte, pela condição socioeconômica, por barreiras culturais e, em alguns casos, pela ausência de profissionais de saúde nos serviços. Dificuldades semelhantes no acesso aos serviços de saúde e também no acesso a bens e serviços em geral foram reveladas em um estudo com populações quilombolas em Minas Gerais 46 e em Goiás 47. Embora existam comunidades quilombolas em áreas urbanas e periurbanas, a grande maioria delas encontra-se na zona rural 25 . As diferenças entre o rural e o urbano no Brasil são evidentes nos indicadores educacionais e de saúde 14,16,48. Esse dado ajuda a compreender a média de idade mais elevada das participantes deste estudo, pois muitas mulheres deixam as comunidades em busca de melhores condições de vida e de um maior acesso aos serviços de saúde e educação.

A prevalência de $\operatorname{HIV}(0,3 \%)$ nas mulheres deste estudo foi baixa e semelhante à encontrada em gestantes indígenas no Brasil, como descrito no estudo de revisão sistemática 49 sobre HIV, sífilis e hepatite viral em afrodescendentes e indígenas da América Latina. Porém, prevalência de infecção pelo HIV mostrou-se mais elevada $(0,11 \%)$ entre as mulheres indígenas não gestantes em outra região da Amazônia 50. Vale ressaltar que a notificação compulsória dos casos de HIV no Brasil teve início no ano de 201451.

A infecção pelo HIV é considerada um dos maiores problemas de saúde pública em todo o mundo 52. No ano de 2018, a Região Sudeste apresentou o maior número de casos novos de HIV no país, com 37,7\% dos casos notificados no Sistema de Informação de Agravos de Notificação (SINAN). No período de janeiro de 2007 a junho de 2019, 49,7\% dos casos ocorreram entre pretos e pardos 49 . Esse fato poderá ser considerado pelos gestores de saúde da região do presente estudo na implementação de políticas públicas direcionadas aos povos quilombolas, facilitando-lhes o acesso aos serviços de saúde, a oferta de teste rápido para o HIV e tratamento oportuno nos centros especializados, evitando a transmissão de 53 novos casos na comunidade e diminuindo as iniquidades em saúde.

Neste estudo, a prevalência por sífilis foi superior a 4\%. Considera-se que é uma prevalência elevada, se comparada à de 2,9\% no Estado do Espírito Santo, no ano de 2018 53. Uma prevalência ainda mais elevada, maior que 5\%, foi detectada em comunidades indígenas do Paraguai e do Peru, em um estudo de revisão sistemática 49 . No entanto, prevalência menor $(1,51 \%)$ foi encontrada em mulheres indígenas da Amazônia 50 e em mulheres jovens (0,15\%) no Centro-oeste do Brasil 39.

A prevalência elevada de sífilis nas mulheres quilombolas incluídas no estudo torna-se preocupante, uma vez que a doença tem como uma das suas repercussões o aumento do risco de transmissão materno-fetal e complicações. Como é difícil o acesso aos serviços de saúde para essas mulheres, possivelmente, na ocorrência de uma gestação, o pré-natal ficará prejudicado, contribuindo para manutenção das altas taxas da sífilis congênita, de outras morbidades, de risco de outras IST e até mesmo de óbito neonatal.

O consumo de álcool, resultado alterado no exame citopatológico e detecção de vaginose bacteriana foram os fatores independentes associados às IST nas mulheres quilombolas. O uso do álcool pode reduzir a percepção de risco para as IST e a capacidade de negociar o uso do preservativo com o parceiro sexual 54. Em mulheres jovens afroamericanas 54,55, o consumo de álcool aumentou os com- 
portamentos sexuais de risco, inclusive com aumento do número de parceiros sexuais e infecção por IST. Devido à ausência de políticas públicas de lazer e de cultura direcionadas às populações de área rural, desde muito jovens, homens e mulheres quilombolas são expostos ao consumo do álcool 56,57, tornando essas mulheres ainda mais expostas às IST.

No que se refere ao resultado do exame citopatológico, a prevalência geral de alterações no exame neste estudo $(7,7 \%)$ foi semelhante à detectada em mulheres de duas distintas comunidades quilombolas da região Nordeste, que foi de $8 \% 23$ e 8,4\% 36. Mulheres negras têm um risco aumentado para um diagnóstico tardio do câncer de colo de útero 45,58. Logo, o acesso precário aos serviços de saúde pelas mulheres quilombolas, como as longas distâncias a serem percorridas, faz com que o exame citopatológico alterado se torne um problema relevante, fato que poderá repercutir no diagnóstico tardio do câncer de colo de útero.

Outra questão importante é o diagnóstico de vaginose bacteriana como fator associado independente para IST nas mulheres quilombolas. Essa é uma infecção genital frequente nas mulheres 10,59. A maior parte dos casos é assintomática 59 e há aumento do risco de infecção por C. trachomatis e $N$. gonorrhoeae 60 e de outras IST 61. Assim, o manejo adequado e oportuno das infecções genitais pelos profissionais de saúde do serviço oferece benefícios à qualidade de vida, como também à saúde sexual e reprodutiva da mulher quilombola e do seu(s) parceiro(s), contribuindo com a redução de novos casos de IST na comunidade. O fato de a maior parte das mulheres quilombolas deste estudo residir em área rural pode refletir em uma baixa utilização do sistema de saúde local, dado que mais da metade das quilombolas que tiveram IST detectada relatou que é difícil a impossível o acesso à unidade básica de saúde nos municípios estudados (Tabela 2).

Devido à dificuldade de acesso aos serviços de saúde, é comum o uso de terapias alternativas (medicamentos caseiros) pelas comunidades quilombolas. O saber tradicional transmitido a cada geração, como as rezas, o uso de xaropes, emplastos e chás para tratamento de algumas doenças, é frequente e relatado em estudos 47,62 com comunidades quilombolas de Goiás e Minas Gerais. As terapias alternativas no manejo das doenças são usuais em outras comunidades tradicionais, como as ribeirinhas e indígenas, e são empregada pela população antes mesmo de recorrer ao sistema biomédico-hegemônico e em conjunto com medicamentos alopáticos 47,63. O conhecimento dessas práticas tradicionais é fundamental durante a formação dos profissionais de saúde e para os que atuam principalmente na atenção primária à saúde 63 para promover um cuidado à saúde efetivo e culturalmente aceito 64 .

Podemos citar como limitação do estudo um potencial viés de informação, dada a baixa frequência de respostas positivas para os fatores de risco para as IST, devido às respostas relacionadas aos comportamentos sexuais íntimos ocorridos ao longo da vida. A própria característica do estudo transversal de medir a exposição e o desfecho ao mesmo tempo não permite estabelecer uma relação de causa e efeito. Além disso, a falta de um banco de dados municipal e a ausência de vínculo dessas mulheres a algum serviço público de assistência à saúde, educação ou assistência social dificultaram o recrutamento por grupo de domicílios e por faixa etária. Geograficamente, as residências são muito afastadas uma das outras, o que dificultou o contato com algumas mulheres e a coleta de dados do estudo. Essa limitação pode ter subestimado a prevalência do desfecho na amostra estudada, entretanto acredita-se que a alta taxa de aceite na participação do estudo tenha diminuído esse problema.

Este foi o primeiro estudo no Estado do Espírito Santo que apresentou um panorama inédito das IST em mulheres de comunidades quilombolas, e pode também revelar as diversas vulnerabilidades sociais e de saúde 14 vivenciadas por essas mulheres. Em sua maioria, elas residem em área rural, distantes do centro urbano, com difícil acesso aos serviços de saúde, têm renda baixa, baixa escolaridade e acesso restrito a bens e serviços em geral. Essas condições tornam a mulher quilombola ainda mais vulnerável às IST. Também sofrem desigualdades nas relações e violência em função de gênero, raça e classe social 65 .

Uma vez identificadas as fragilidades, a exposição para as IST e os fatores associados, e dada a importância das infecções na saúde sexual e reprodutiva das mulheres, este estudo poderá contribuir com a implementação de políticas públicas de saúde baseadas em evidências, direcionadas a uma população específica, em cumprimento à Política Nacional de Atenção Básica (PNAB) 66 e à PNSIPN 67.

Além disso, este estudo contribuirá com os gestores de saúde, para a qualificação e sensibilização dos profissionais nos serviços de saúde, na atenção à saúde da mulher negra, para o rastreamento e 
tratamento oportuno das mulheres quilombolas e seus parceiros sexuais que vivem distantes da área urbana e com difícil acesso aos serviços de saúde, reduzindo as iniquidades e enfrentando o racismo institucional, ainda vigente em nossa sociedade.

Pode-se concluir que a prevalência geral de IST foi elevada nas mulheres quilombolas, caracterizadas pela baixa escolaridade e pela dificuldade de acesso aos serviços de saúde. As mulheres quilombolas com idade entre 25 e 44 anos, consumidoras de álcool, com exame citopatológico alterado, além de vaginose bacteriana, apresentaram uma chance maior de serem diagnosticadas com um ou mais agentes de transmissão sexual.

\section{Colaboradores}

J. A. Dias contribuiu com a concepção, execução da pesquisa, interpretação dos dados e redação do manuscrito. T. V. Luciano, M. C. L. F. S. Santos e C. Musso contribuíram com a execução da pesquisa e revisão crítica do estudo e da versão final do manuscrito. E. Zandonade contribuiu com o delineamento do estudo e análise e interpretação dos dados. L. C. Spano contribuiu com a revisão crítica do estudo e da versão final do manuscrito. A. E. Miranda contribuiu com a concepção e delineamento do estudo, execução da pesquisa e análise e interpretação dos dados.

\section{Informações adicionais}

ORCID: Jerusa Araujo Dias (0000-0002-04902488); Thaís Verly Luciano (0000-0003-3791-3762); Maria Carmen Lopes Ferreira Silva Santos (00000003-4564-1859); Carlos Musso (0000-0003-49281280); Eliana Zandonade (0000-0001-5160-3280); Liliana Cruz Spano (0000-0002-6205-6988); Angelica Espinosa Miranda (0000-0002-5556-8379).

\section{Agradecimentos}

Ao Conselho Nacional de Desenvolvimento Científico e Tecnológico e ao Fundo de Amparo à Pesquisa e Inovação do Espírito Santo pelo financiamento.

\section{Referências}

1. World Health Organization. Global incidence and prevalence of selected curable sexually transmitted infections-2008. Geneva: World Health Organization; 2012.

2. Korenromp EL, Rowley J, Alonso M, Mello MB, Saman Wijesooriya N, Guy Mahiané S, et al. Global burden of maternal and congenital syphilis and associated adverse birth outcomes - estimates for 2016 and progress since 2012. PLoS One 2019; 14:e0211720.

3. HPV Information Centre. Statistics. http:// www.hpvcentre.net/datastatistics.php\% 0Apapers2://publication/uuid/64881A96BF55-4B84-920C-D127A34FB036 (acessado em 24/Jun/2019).

4. World Health Organization; Joint United Nations Programme on HIV/AIDS. Summary global HIV epidemic (2017). Geneva: World Health Organization; 2018.

5. Gomez GB, Kamb ML, Newman LM, Mark J, Broutet N, Hawkes SJ. La sífilis materna no tratada y los resultados adversos en el embarazo: revisión sistemática y metanálisis. Bull World Health Organ 2013; 91:217-26.

6. Silver BJ, Guy RJ, Kaldor JM, Jamil MS, Rumbold AR. Trichomonas vaginalis as a cause of perinatal morbidity: a systematic review and meta-analysis. Sex Transm Dis 2014; 41:36976.

7. Fichorova RN. Impact of T. vaginalis infection on innate immune responses and reproductive outcome. J Reprod Immunol 2009; 83:185-9.

8. Hou R, Xu C, Zhang S, Wu M, Zhang W. Distribution of human papillomavirus genotype and cervical neoplasia among women with abnormal cytology in Beijing, China. Int J Gynecol Obstet 2012; 119:257-61.

9. Toyer AL, Trignol-Viguier N, Mereghetti L, Joly B, Blin E, Body G, et al. Interest of simultaneous Chlamydia trachomatis and Neisseria gonorrhoeae screening at the time of preabortion consultation. Contraception 2012; 86:572-6.

10. Kenyon C, Colebunders R, Crucitti T. The global epidemiology of bacterial vaginosis: a systematic review. Am J Obstet Gynecol 2013; 209:505-23. 
11. Aaron E, Blum C, Seidman D, Hoyt MJ, Simone J, Sullivan M, et al. Optimizing delivery of HIV preexposure prophylaxis for women in the United States. AIDS Patient Care STDS 2018; 32:16-23.

12. Hess KL, Hu X, Lansky A, Mermin J, Hall HI. Lifetime risk of a diagnosis of HIV infection in the United States. Ann Epidemiol 2017; 27:238-43.

13. Hay P. Bacterial vaginosis. F1000Res 2014; 6:59-63.

14. Prestes CRS, Paiva VSF. Abordagem psicossocial e saúde de mulheres negras: vulnerabilidades, direitos e resiliência. Saúde Soc 2016; 25:673-88.

15. Werneck J. Racismo institucional e saúde da população negra. Saúde Soc 2016; 25:535-49.

16. Gomes KO, Reis EA, Guimarães MDC, Cherchiglia ML. Utilização de serviços de saúde por população quilombola do Sudoeste da Bahia, Brasil. Cad Saúde Pública 2013; 29:1829-42.

17. Benjamins MR, Whitman S. Relationships between discrimination in health care and health care outcomes among four race/ethnic groups. J Behav Med 2014; 37:402-13.

18. Swartzendruber A, Sales JM, Brown JL, Davis TL, Diclemente RJ, Rose E. Predictors of repeat Chlamydia trachomatis and/or Neisseria gonorrhoeae infections among African-American adolescent women. Sex Transm Infect 2013; 89:76-82.

19. Ibrahim S, Bukar M, Galadima G, Audu B, Ibrahim H. Prevalence of bacterial vaginosis in pregnant women in Maiduguri, North-Eastern Nigeria. Niger J Clin Pract 2014; 17:154-8.

20. Maina AN, Kimani J, Anzala O. Prevalence and risk factors of three curable sexually transmitted infections among women in Nairobi, Kenya. BMC Res Notes 2016; 9:193.

21. Araújo EM, Costa MCN, Noronha CV, Hogan VK, Vines AL, Araújo TM. Desigualdades em saúde e raça/cor da pele: revisão da literatura do Brasil e dos Estados Unidos (1996-2005). Saúde Coletiva 2010; 7:116-21.

22. Dias ICC, Nascimento MDSB, Batista JE, Vidal FCB, Silva DF, Silva MACN, et al. Câncer de colo do útero, genotipagem do Papiloma Vírus Humano (HPV) em mulheres quilombolas de um município brasileiro: aceitabilidade da vacina. Cad Pesqui (São Luís) 2014; 21 (especial):1-11.

23. Batista JE, Monteiro SG, Moraes OKDN, Batista-Filho JE, Lobão WJM, Santos GBS, et al. Fatores associados ao vírus HPV e lesões cervicais em mulheres quilombolas. Revista de Pesquisa em Saúde 2014; 15:218-22.

24. Instituto Nacional do Câncer José Alencar Gomes da Silva. Nomenclatura brasileira para laudos citopatológicos cervicais. Rio de Janeiro: Instituto Nacional do Câncer José Alencar Gomes da Silva; 2012.

25. Fundação Cultural Palmares. Informações quilombolas. http://www.palmares.gov.br/?pa ge_id=52126 (acessado em 18/Jun/2019).
26. Ministério da Saúde. Sistema de Informação do câncer do colo do útero e Sistema de Informação do câncer de mama. http://w3.datasus. gov.br/siscam/index.php?area $=02$ (acessado em 10/Set/2018).

27. Victora CG, Huttly SR, Fuchs SC, Olinto MT. The role of conceptual frameworks in epidemiological analysis: a hierarchical approach. Int J Epidemiol 1997; 26:224-7.

28. Aziz S, Sweat D. Subsequent HIV diagnosis risk after syphilis in a Southern Black population. Sex Transm Dis 2018; 45:643-7.

29. Benzaken A, Sabidó M, Galban E, Rodrigues Dutra DL, Leturiondo AL, Mayaud P. HIV and sexually transmitted infections at the borderlands: situational analysis of sexual health in the Brazilian Amazon. Sex Transm Infect 2012; 88:294-300.

30. Luppi CG, Oliveira RLS, Veras MA, Lippman $\mathrm{SA}$, Jones $\mathrm{H}$, Jesus $\mathrm{CH}$, et al. Diagnóstico precoce e os fatores associados às infecções sexualmente transmissíveis em mulheres atendidas na atenção primária. Rev Bras Epidemiol 2011; 14:467-77.

31. Wohlmeister D, Vianna DRB, Helfer VE, Gimenes F, Consolaro MEL, Barcellos RB, et al. Association of human papillomavirus and Chlamydia trachomatis with intraepithelial alterations in cervix samples. Mem Inst Oswaldo Cruz 2016; 111:106-13.

32. Barcelos MRB, Vargas PRM, Baroni C, Miranda AE. Infecções genitais em mulheres atendidas em Unidade Básica de Saúde: prevalência e fatores de risco. Rev Bras Ginecol Obstet 2008; 30:349-54.

33. Ministério da Saúde. Prevalências e freqüências relativas de doenças sexualmente transmissíveis (DST) em populações selecionadas de seis capitais brasileiras, 2005. Brasília: Ministério da Saúde; 2008.

34. Fernandes LB, Arruda JT, Approbato MS, García-Zapata MTA. Infecção por Chlamydia trachomatis e Neisseria gonorrhoeae: fatores associados à infertilidade em mulheres atendidas em um serviço público de reprodução humana. Rev Bras Ginecol Obstet 2014; 36:353-8.

35. Boldrini NT, Freitas LB, Coutinho AR, Loureiro FZ, Spano LC, Miranda AE. High-grade cervical lesions among women attending a reference clinic in Brazil: associated factors and comparison among screening methods. PLoS One 2014; 9:e102169.

36. Nascimento MDSB, Vidal FCB, Silva MACN, Batista JE, Lacerda Barbosa MC, Muniz Filho WE, et al. Prevalence of human papillomavirus infection among women from quilombo communities in northeastern Brazil. BMC Womens Health 2018; 18:1.

37. Ministério da Saúde. Portaria no 207, de 17 de fevereiro de 2016. Lista Nacional de Notificação Compulsória. Diário Oficial da União 2016; 18 fev.

38. Rocha DA, Filho RA, Mariño JM, Santos CM. "Hidden" sexually transmitted infections among women in primary care health services, Amazonas, Brazil. Int J STD AIDS 2014; 25:878-86. 
39. Lima YAR, Turchi MD, Fonseca ZC, Garcia FLB, Brito e Cardoso FA, Guarda Reis MN, et al. Sexually transmitted bacterial infections among young women in Central Western Brazil. Int J Infect Dis 2014; 25:16-21.

40. Lima Soares V, Mesquita AMTS, Cavalcante FGT, Silva ZP, Hora V, Diedrich T, et al. Sexually transmitted infections in a female population in rural north-east Brazil: prevalence, morbidity and risk factors. Trop Med Int Health 2003; 8:595-603.

41. Lin L, Benard VB, Greek A, Hawkins NA, Roland KB, Saraiya M. Racial and ethnic differences in human papillomavirus positivity and risk factors among low-income women in Federally Qualified Health Centers in the United States. Prev Med (Baltim) 2015; 81:258-61.

42. Sanjosé S, Diaz M, Castellsagué X, Clifford G, Bruni L, Muñoz N, et al. Worldwide prevalence and genotype distribution of cervical human papillomavirus DNA in women with normal cytology: a meta-analysis. Lancet Infect Dis 2007; 7:453-9.

43. Vidal AC, Smith JS, Valea F, Bentley R, Gradison M, Yarnall KSH, et al. HPV genotypes and cervical intraepithelial neoplasia in a multiethnic cohort in the southeastern USA. Cancer Causes Control 2014; 25:1055-62.

44. Yoo W, Kim S, Huh WK, Dilley S, Coughlin SS, Partridge EE, et al. Recent trends in racial and regional disparities in cervical cancer incidence and mortality in United States. PLoS One 2017; 12:e172548.

45. Thuler LCS, Aguiar SS, Bergmann A. Determinantes do diagnóstico em estadio avançado do câncer do colo do útero no Brasil. Rev Bras Ginecol Obstet 2014; 36:237-43.

46. Marques AS, Freitas DA, Leão CDA, Oliveira SKM, Pereira MM, Caldeira AP. Atenção primária e saúde materno-infantil: a percepção de cuidadores em uma comunidade rural quilombola. Ciênc Saúde Colet 2014; 19:365-71.

47. Santos RC, Silva MS. Condições de vida e itinerários terapêuticos de quilombolas de Goiás. Saúde Soc 2014; 23:1049-63.

48. Castro CN, Pereira CN. Educação: contraste entre o meio urbano e o meio rural no Brasil. Bol Reg Urbano Ambient 2019; (21):63-74.

49. Russell NK, Nazar K, del Pino S, Gonzalez MA, Bermúdez XPD, Ravasi G. HIV, syphilis, and viral hepatitis among Latin American indigenous peoples and Afro-descendants: a systematic review. Rev Panam Salud Pública 2019; 43:e17.

50. Benzaken AS, Sabidó M, Brito I, Bermúdez XPD, Benzaken NS, Galbán E, et al. HIV and syphilis in the context of community vulnerability among indigenous people in the Brazilian Amazon. Int J Equity Health 2017; 16:92.

51. Secretaria de Vigiância em Saúde, Ministério da Saúde. Boletim Epidemiológico HIV/Aids 2019. Brasília: Ministério da Saúde; 2019.

52. World Health Organization. Data and statistics: HIV/AIDS. https://www.who.int/hiv/ data/en/ (acessado em 07/Mai/2019).
53. Secretaria de Vigilância em Saúde, Ministério da Saúde. Boletim Epidemiológico Sífilis 2019. Brasília: Ministério da Saúde; 2019.

54. Seth P, Wingood GM, DiClemente RJ, Robinson LS. Alcohol use as a marker for risky sexual behaviors and biologically confirmed sexually transmitted infections among young adult African-American women. Womens Health Issues $2011 ; 21: 130-5$.

55. Fisher JC, Cook PA, Sam NE, Kapiga SH. Patterns of alcohol use, problem drinking, and HIV infection among high-risk African women. Sex Transm Dis 2008; 35:537-44.

56. Sousa C, Santos RS, Santana KC, Souzas R, Leite ÁJM, Medeiros DS. Sexual behavior and associated factors in rural adolescents. Rev Saúde Pública 2018; 52:39.

57. Partelli ANM, Cabral IE. Stories about alcohol drinking in a quilombola community: participatory methodology for creating-validating a comic book by adolescents. Texto Contexto Enferm 2018; 26:e2820017.

58. Fleming S, Schluterman NH, Tracy JK, Temkin SM. Black and white women in Maryland receive different treatment for cervical cancer. PLoS One 2014; 9:e104344.

59. Bautista CT, Wurapa EK, Sateren WB, Morris SM, Hollingsworth BP, Sanchez JL. Association of bacterial vaginosis with chlamydia and gonorrhea among women in the U.S. Army. Am J Prev Med 2017; 52:632-9.

60. Wijgert JHHM, Borgdorff H, Verhelst R, Crucitti T, Francis S, Verstraelen H, et al. The vaginal microbiota: what have we learned after a decade of molecular characterization? PLoS One 2014; 9:e105998.

61. Nardis C, Mosca L, Mastromarino P. Vaginal microbiota and viral sexually transmitted diseases. Ann Ig 2013; 25:443-56.

62. Freitas DA, Silveira JCS, Ferreira LA, Zucchi P, Marques AS. Mulheres quilombolas: profissionais na Estratégia de Saúde da Família. Espaç Saúde (Online) 2011; 12:56-62.

63. Langdon EJ, Wiik FB. Antropologia, saúde e doença: uma introdução ao conceito de cultura aplicado às ciências da saúde. Rev Latinoam Enferm 2010; 18:173-81.

64. Marrone SR. A case for transcultural nursing administration. J Healthc Commun 2018; 3:16.

65. Secretaria de Gestão Estratégica Participativa, Ministério da Saúde. Tecendo a saúde das mulheres do campo, da floresta e das águas: direitos e participação social. Brasília: Ministério da Saúde; 2015.

66. Ministério da Saúde. Portaria no 2.436, de 21 de setembro de 2017. Aprova a Política Nacional de Atenção Básica, estabelecendo a revisão de diretrizes para a organização da Atenção Básica, no âmbito do Sistema Único de Saúde (SUS). Diário Oficial da União 2017; 22 set.

67. Secretaria Especial de Políticas de Promoção da Igualdade Racial. Política Nacional de Saúde Integral da População Negra. Brasília: Ministério da Saúde; 2017. 


\section{Abstract}

The study aimed to estimate the prevalence of sexually transmissible infections (STIs) and associated factors in women in quilombola (maroon) communities in Brazil. This was a populationbased cross-sectional study of quilombola women from March 2017 to January 2019. A questionnaire was used with sociodemographic, behavioral, and clinical information. A gynecological examination was performed for the collection of uterine cervical cells for oncotic cytology and the detection of Chlamydia trachomatis, Neisseria gonorrhoeae, Trichomonas vaginalis, and human papillomavirus (HPV) via polymerase chain reaction. Rapid tests for HIV and syphilis were performed. The main outcome was defined as infection with one or more sexually transmissible pathogens. The analysis used chi-square test and hierarchical logistic regression. From a total of 380 women, 352 (92.6\%) were included in the study. Prevalence of at least one STI was 18.5\% (95\%CI: 14.76-22.85). The highest prevalence was for $\mathrm{HPV}$, with $11.1 \%$, followed by $6.3 \%$ for $\mathrm{T}$. vaginalis and $4.3 \%$ for $\mathrm{C}$. trachomatis. There were no cases of $\mathrm{N}$. gonorrhoeae. Prevalence was 0.3\% for HIV and $4.3 \%$ for syphilis. Cervical-vaginal cytology was altered in $7.7 \%$ of the women. Detection of one or more STIs was significantly associated with age 25 to 44 years $(O R=2.33$; 95\%CI: 1.05-5.18), alcohol consumption (OR = 1.96; 95\%CI: 1.06-3.64), altered cervical cytology $(O R=3.96 ; 95 \% C I: 1.65-9.48)$, and bacterial vaginosis (OR = 3.61; 95\%CI: 2.01-6.47). Quilombola women showed high prevalence of one or more STIs, emphasizing the importance of organizing prevention strategies targeted to these women.

Sexually Transmitted Diseases; Women; Cross-Sectional Studies

\section{Resumen}

El objetivo del estudio fue estimar la prevalencia de enfermedades de transmisión sexual (ETS) y sus factores asociados en mujeres quilombolas en Brasil. Estudio transversal de base poblacional con mujeres quilombolas durante el período de marzo de 2017 a enero de 2019. Se utilizó un cuestionario con información sociodemográfica, comportamental y clínica. Se realizó un examen ginecológico, a fin de recoger células cervicales para la citología oncótica, así como para la detección de Chlamydia trachomatis, Neisseria gonorrhoeae, Trichomonas vaginalis, $y$ virus del papiloma humano (VPH) mediante un test de reacción en cadena de la polimerasa. Se realizó un test rápido para VIH $y$ sífilis. El resultado principal, se definió como infección por uno o más agentes infecciosos de transmisión sexual. Para el análisis, se utilizó el test de chi-cuadrado y regresión logística jerárquica. De un total de 380 mujeres, 352 (92,6\%) se incluyeron en el estudio. La prevalencia de por lo menos una ETS fue de 18,5\% (IC95\%: 14,76-22,85). La mayor prevalencia fue por VPH 11,1\%, seguida de $6,3 \%$ por $\mathrm{T}$. vaginalis $y$ 4,3\% por $\mathrm{C}$. trachomatis. No hubo casos de N. gonorrhoeae. Para el VIH, la prevalencia fue de 0,3\% y de sifilis fue de 4,3\%. La citología cérvico-vaginal estaba alterada en un 7,7\%. La detección de una o más ETS estuvo significativamente asociada con la edad entre 25 a 44 años $(O R=2,33$; IC95\%: 1,05-5,18), el consumo de alcohol $(O R=1,96$; IC95\%: 1,06-3,64), resultado alterado de la citología $(O R=3,96$; IC95\%: $1,65-9,48)$ y vaginosis bacteriana $(O R=3,61$; IC95\%: 2,01-6,47). Las mujeres quilombolas presentaron una elevada prevalencia por una o más ETS, por lo que es importante la elaboración de estrategias de prevención dirigidas a estas mujeres.

Enfermedades de Transmisión Sexual; Mujeres; Estudios Transversales
Recebido em 10/Set/2019

Versão final reapresentada em 04/Jun/2020

Aprovado em 29/Jun/2020 